Review

\title{
Eye-tracking in immersive virtual reality for education: a review of the current progress and applications
}

\begin{abstract}
The concept of using eye-tracking in virtual reality for education has been researched in various fields over the past years. With this review, we aim to discuss the recent advancements and applications in this area, explain the technological aspects, highlight the advantages of this approach and inspire interest in the field. Eye-tracking has already been used in science for many decades and now has been substantially reinforced by the addition of virtual and augmented reality technologies. The first part of the review is a general overview of eye-tracking concepts and its applications. In the second part, the focus shifted towards application of eye-tracking in virtual reality. The third part is the description of the recently emerged concept of eye-tracking in virtual reality when applied to education and studying, which has not been thoroughly described before. We describe the main findings, technological aspects and advantages of this approach.
\end{abstract}

Keywords: eye-tracking; virtual reality; education and VR, education and eyetracking

\section{Introduction}

Eye-tracking is a technology that has been for a long time used in humanitarian and medical research [1,2], and quite recently it has become a useful tool in the field of user experience design. The most well-known and cited example of such eye-tracking use is a study from 2006, which showed that while people browse the internet and look at the search engine results, their attention is scattered on the page in a shape of a triangle or letter $F$, this research has then been followed up and proved in the more recent years [3]. Active use of eye-tracking technology has facilitated the delivery of information to the consumer in the media. Over time, the technology was transposed into biomedical research and showed good results in the rehabilitation measures after ischemic brain damage [4-10].

The successful use of technology in treatment has prompted researchers to pay attention to the prospects of its use in studying healthy people. Thus, when studying the decision-making process by an individual about the need for social interactions in a virtual environment, it was demonstrated that eye-tracking technologies can be used as a forecasting tool. The frequency and duration of fixation, the peak velocity and amplitude of saccades can be used to identify and eliminate transient states of uncertainty when making perceptual decisions [11], as well as to assess the complexity of the problem when it becomes too complicated due to overloading with details [12]. In predicting decision making, gaze fixation, fixation duration, and the number and duration of visits are of great importance. Sustainable 
combinations of these parameters form various starring strategies [13].

Also, with the help of eye-tracking it is possible to verify other higher nervous functions: emotions, regret and disappointment [14], memorization processes [15]. In addition to the prognostic function, eye-tracking technologies can be used to control decision-making [16] and, in particular, to increase its value [17]. As one of the possible options for such an impact on the adoption of a two-alternative forced choice of a test subject, a simple adjustment of the decision time can be used with interruption at the moment of fixing the gaze on the target option [18]. An important study has demonstrated [19] that the number of eye movements between informationally important objects in the context of the current task is an indicator that is inversely related to the quality of solving a cognitive task.

Research in the field of analysis of eye movements and eye trajectories in the context of virtual three-dimensional space is fairly new. Some works are aimed at studying the emotional behavior of a person interacting with objects in a virtual space, in particular in studies [20,21], implicit eye movements and their significance were analyzed with regards to the influence of attention on the emotional learning processes. Some researchers are studying the possibility of forming interactive interfaces for interacting with a virtual environment by changing the trajectory of the gaze [22]. Research in the field of education is also beginning to appear, for example, in [23], the possibility of constructing a predictive system of the level of students' skills was studied by analyzing their trajectory of view in a virtual environment. Part of the research is also aimed at analyzing the usability of VR interfaces by studying the gaze trajectory fixed in virtual three-dimensional space [24]. Thus, over the past 5 years, sufficient experience has been gained in using eye-tracking as a technology for predicting, evaluating and managing the solution of emotional and cognitive tasks.

Although there has been extensive research and many review papers on the basic concepts and applications of the eye-tracking technology to various fields, the concept of eye-tracking in virtual reality for educational purposes is a rather new direction that has only appeared in the last few years. The bases behind it consists of the assumption that if eye-tracking is a helpful tool for enhancing cognitive functions in ill and healthy people, it could also be used to enhance the learning process in students. Hence, this review article is aimed at highlighting the current progress in the area of eye-tracking in virtual reality for educational purposes. The article consists of three parts: brief overview of eye-tracking techniques and application fields with the highlights of major developments, the implementation of eye-tracking in virtual reality in different fields of science and the description of on-going new research in the field of eye-tracking in virtual reality and education.

\section{Eye-tracking concepts and applications}


Eye-tracking has been broadly used for many years as a tool for investigation of human cognition. It first appeared in the early 20th century, when special contact lenses were used with a pointer attached to them as a first eye-tracker device. Later this technique has been changed and optimised by using light beams and recording their reflection on film instead $[25,26]$. In the 1960 's the eye-tracking as we know it now have been developed, new approaches have evolved and since then have been further studied and improved $[27,28]$. It is safe to say that the methods used for detecting eye-movements have become significantly more accurate in the recent years. At the moment, the most used methods in the field of eyetracking involve the video systems with computer vision techniques usage [29]. With the advances in the field of optics and microelectronics, the emergence of high-quality cameras, made it possible to use eye-tracking devices of small size, which can even be fitted to the portable "smart" glasses or a VR headset. These facilitate accurate measure of eye movement and provide large amounts of data. These eye tracking methods have been described in a variety of different experimental methods [30]. These technological advances can explain the fact that eye-tracking is now widely and efficiently used in human cognition research.

\subsection{Technological aspects}

The concept of modern eye tracking device is based on an array of infrared or near-infrared light sources and cameras that track the gaze behavior of one or both eyes [31]. In most of the currently used systems, an array of invisible light sources illuminates the eye and produces a corneal reflection, then the eye tracker device records the relationship between the centre of the pupil and the reflection, on order to calculate the vectors that can fully describe the relationship of eye position to the location in the perceived world [29]. The calculated viewpoint in space moves with the movement of eyes. At the moment, eye trackers are available in several hardware configurations, varying in size and price. These include more conventional "chin-up" systems for head stabilization, remote systems that can only account for a limited amount of head movement and mobile eyewear-based systems, the latter being the newest type. All of these hardware types have different advantages and disadvantages that are related to spatial (i.e. tracking) accuracy, tracking speed, mobility, portability, and cost $[32,33]$.

The indicators of visual behaviour that are important for the understanding of the interpretive process and are provided by eye-trackers include: motion, position, numeracy and latency indicators [31]. In order to get a clear picture of how eye-trackers collect data, it is important to realize that the eye is constantly moving between fixation points. The fixations are instantaneous pauses of the eyes in space for a very short time of time (milliseconds). The movements between successive fixations are called saccades [31]. Motion measurements provide quantitative analyses of the 
eye movement patterns in space during saccades, for example the distance between successive saccades and saccade speed. The position of the gaze is measured in Cartesian coordinate space which can be done in a real scene or on a computer monitor. Numerical measures are used to identify the frequency of fixations and saccades of the eyes during scene perception, for example per unit time, and how these values change according to the position of the subject. The temporal dynamics of fixations and saccades is then estimated by the latency measures, these can include the duration of first fixation, subsequent fixations and the duration of saccades.

The recorded patterns of sequential saccades and fixations are then analysed with regards to the factors that can influence them. These factors include both internal states, for example, frustration, anxiety, mental load, uncertainty, and external states, such as the salience and organisation of the stimulus.

After explaining the technical side of the eye-tracking process, it is important to consider the areas of science and research where this technology has been most successfully applied in recent years.

\subsection{In behavioral science}

One of the fields where eye-tracking has been extensively used and studied over the last decade is without a doubt behavioral and cognitive science. There have been many studies that present eye-trackers for tracking changes in cognition [34]. In one of such studies it has been shown that the total viewing time and fixation time in areas corresponding to noncooperative solutions is associated with the general level of participants' cooperation. The increase in the total viewing time and fixation time on the areas that correspond to non-cooperative solutions happens due to the preference for non-cooperation by participants and a decrease in the overall level of cooperation. Therefore, the viewing time clearly correlates with the decision making process. The number of fixations on group attributes is associated with group identity, but it does not immediately lead to cooperative behavior [9].

Eye-tracking technology has been a useful tool in the research that is related to social attention. For example, it has been shown that results from certain experimental studies correlate with measures of social impairment and with autism symptom severity. It has been found that reduced attention to social stimuli or increased attention to non-social stimuli is correlated with behavioral measures of autism [35-37]. Face processing as well as language skills are also significantly correlated with measures of social attention. A strong association between face processing skills and attention to faces has been reported for children [38], more studies suggested that attention to a speaker's mouth and eyes could be a predictive measure of how fast the words are recognised among children with ASD [39]. Moreover, when the processing of such social information as eye and mouth movement is atypical, it is correlated with difficulties in language learning or social impairment. An increase in the attention towards mouth has been 
associated with increased social adaptation [40] and communicative competence [41]. These results suggest that eye-tracking methods are promising for studying social attention in ASD and can be successfully used in studies with children.

\subsection{Eye tracking in medicine}

The eye-tracking has been extensively used in medical research. This has been reported and reviewed in many papers over the past decade [2,42-45]. As one of the prior examples, eye-trackers have been used alongside machine learning to improve the diagnostics and predict diagnostics errors before they can even occur. These techniques can provide automatic cueing or feedback to be provided to learners during image examination [46]. The wide use of this technology has become available due to eye trackers becoming more portable, easier to use, cheaper and more available to consumers. With regards to diagnostics, automatic feedback has become possible by parsing medical images into diagnostically relevant and nonrelevant regions (ROIs) by using expert annotation or automated machine vision techniques [47-49]. Once these regions are known to the system that is used eye tracking, fixations on the important regions are recorded. The recordings are then used to study spatial distribution of attention over a digital image and the time of fixations. Once there is enough data available, it can be fed into the machine learning algorithm to provide automated diagnostics.

Another significant area of medical research that is associated with the use of eye-tracking devices is related to rehabilitation. It has been shown that the combination of virtual reality technology and eye-tracking, improves rehabilitation of such brain functions as attention, memory, motor and visual-spatial abilities and speech when compared to traditional therapy methods [2]. Moreover, it has been reported that virtual reality training was able to stimulate the patients' motivation. The universal ability, which is not tied to the nature and specifics of the disease, to increase motivation and demonstrate better results in rehabilitation of lost cognitive functions has been reported. This universal property was demonstrated for brain injuries [50] and neurodegenerative diseases [4,7,8]. This finding allowed to reduce the total hospitalization time of patients while increasing the duration of rehabilitation training [8]. These few examples demonstrate the importance of this technology in the medical field and the applications to medical education will be discussed further.

\subsection{Eye tracking in design}

There has been a variety of works reporting a wide use of eye-tracking technology in various fields of design. The technology has been implemented in graphic design as well as other computer-based visual 
evaluation methods in order to measure the distribution of visual attention over the past decades [51,52]. In a study from 2016 , eye-tracking was used to assess computer-based visual tools, which helps decision-making in order to investigate ecosystem services [53]. It has been suggested that the ability of users to understand ecosystem services can be improved through the use of such decision support systems. Other studies have analyzed the data from eye-trackers such as fixation time, saccade amplitude, gaze paths etc of how people can perceive landscapes [54-56]. The opportunities, as well as challenges for the use of eye-tracking in the fields of cartography, geographic information science, spatial cognitions etc. have recently been reported in a broad review [57]. Eye-tracking has also been investigated in the built environment, for example a study of a contextual guidance model with a Bayesian framework was used in order to predict regions of gaze fixation of people while they search for objects in space (eg. pedestrian paintings on the street) [58]. Similar work has been done by Ehinger et al. [59] with an addition of the pedestrians being present or absent in the picture. Eye-tracking in the built environment has also been used to find out about visual preferences that people have with regards to the general objects in public spaces [60]. The effectiveness of GIS software was studied with eye-tracking when the subjects performed orientation tasks with the help of GIS [61]. This study provided a useful insight into the way people orientate, and the subjects that were successful in the self-localization task, were found to spend more visual attention on objects that provided helpful clues.Recently, real-time eye-tracking systems have been implemented to increase efficiency and quality of interactive graphics applications as well as large scale display systems.

\section{Eye-tracking in VR}

Virtual reality (VR) is a powerful tool which can change the way we work and relax, it can also transform the learning techniques in the near future. New applications of VR are invented every day, so it is safe to say that it could be present in our daily life in a relatively short time. The extent of VR research is rather large and recently many virtual reality systems with eye-tracking have emerged.. A vast part of VR research is aimed at improving user-experience and reducing usability issues, and it is believed that eye-tracking technology can be of assistance in this task [62].

Essentially, eye-tracking gives the ability to identify what the user's gaze is focusing on in the virtual reality environment (VRe). Moreover, VR can be used to change the focus of attention if it will increase the positive outcome of the task. There are techniques to draw attention to certain things in the VRe, which can be used if needed. The success of these techniques can be constantly checked, since the gaze of the user is tracked in real time. The use of eye-tracking in VR can be a helpful asset, not only improving the work of various applications, but also identifying disadvantages of some VRe. In VR, full body motion tracking can be used, so the environment can 
react to the user's movement, action and gaze.

In comparison to the real-world eye-tracking, the one in VR has an advantage of the easier definition of the regions that the user had looked at. These regions of interest can also be identified in time and reconstructed. The experimental set-up with eye-tracking in virtual reality is much more flexible and promising for many fields of science since it can be thoroughly controlled. One can control the data collection, environmental settings, and make the stimuli more natural for the user, therefore enhancing the possibilities of research. It is especially useful in research that focuses on human cognition and behaviour. It has been reported that such eyemovement signals as pupil diameter can be used for emotional recognition, and therefore there is a correlation between various emotions and the pupil size [75]. Research has provided a comparison between eye-tracking in VRe emotional recognition and the classic EEG approach. This research suggests that although the eye-tracking classification method was not as efficient as EEG, it still had statistically good enough results to be considered a useful tool for this task. [76].

Various implementations of VR and eye tracking have been numerously reported in the field of computer science. Some research includes very detailed technical reports of VR and eye-tracking implementations. For example a review paper by $\mathrm{V}$. Clay at al gives a step by step explanation of the available hardware and techniques used to implement eye tracking into the VR set [62]. Other studies have been dedicated to the development of VR devices and improvement of existing hardware. There is a particularly interesting study, which assesses what technical requirements a VR set needs to have in order to generate a well-known immersive effect of being "in reality", or as researchers refer to it, in "presence" [63].

A lot of recent VR research is being conducted in the area of computergaming. $\mathrm{T}$ The ability to use eye-gaze in the gameplay has for a long time been a question of investigation and a desired feature for the gaming industry. This topic was given further rise with the launch of the first VR headset with built-in eye-tracking "Fove" in 2016. Apart from using gaze as an instrument of gameplay, eye-tracking can also collect data on where the attention of the gamer is scattered in 3D VRe, therefore, an adaptive game mechanism can create reactive content in the gameplay. One of the papers has shown that the use of gaze to interact with remote objects in the VRe is much faster than the use of hands [64], which could be used as a great advantage for shooting or racing games or any other applications where special attention is paid to speed and aiming quality.

Gaze can also be used as means of nonverbal communication, especially useful in the collaborative VR environments. There has been research showing that the use of VR can actually regulate the interaction between people in this process [65]. The integration of eye-movement in avatar interactions is actively researched with the use of eye-tracking [66]. 
In the area of medical research VR technology has been studied and integrated into the educational process. An example of this would be the study of nurse education in a collaborative immersive system [67], or another research on the medical training in a virtual hospital and medical professional training $[68,69]$. $\mathrm{Vr}$ technology has been reported and researched in dental medicine, such as simulated caries removal exercise for dental students in VR [70] or a surgical education system where the finger tracking is used to show the students the location of the fingers and the exact movements of the expert's fingers during surgery [71]. Another use of VR in medicine is rehabilitation, where the technology is used on patients. Many papers regarding rehabilitation have been reported so fat, an example is a VR based therapy for vestibular problems [72], VRe breathing exercises for people with Chronic Obstructive Pulmonary Disease [73]. VR has been used to visualize the body and actually move through the neural tissue, this feature has been used in medical research [74]. In recent years VR with eye-tracking has been appearing in the medical field. It has proven itself especially useful in the area of medical education and will be described below.

Here we have described the major areas of eye-tracking in VR research which has been rapidly developing in the last years. With the development of this scientific area, more and more research emerges on the advantages and importance of VR in educational programs. This will be discussed in more detail in the next section.

\section{Eye-tracking in VR for education}

\subsection{Overview of recent research and applications}

A concept that has been the major interest of this review is the eyetracking in VR, that can be used in education in order to enhance the learning process and to assess the knowledge of students. This idea has been reported in some literature over the past decade, especially with regards to medical education, however the field is still evolving and the wide use in the other fields is yet to be seen. Here, we describe the findings of the aforementioned papers and suggest ways in which the technology can be applied to the assessment of knowledge in the classroom.

An immersive education environment can potentially provide functionality for assessment procedures with minimal distraction. These technologies can both measure the knowledge level of the taken course and take into account individual aspects, cognitive abilities and achievements of each particular student, therefore personalizing the learning trajectories to enable the best possible result.

Some research has recently been conducted in the area of knowledge assessments, for example, students were asked to read a text or a fragment of text being in the virtual reality environment (VRe). It has been stated that 
their knowledge in a particular field can be judged by data collected from eye-tracker, more specifically, the concentration on specific words and expressions during the task [77].

Eye-tracking has been used for years in medical education and training, and it is believed that eye tracking in VR will provide even more opportunities to enhance the learning process. One of the major findings in this field suggests that experts and novices move their eyes differently [78]. It has been mentioned that the use of eye-tracking in medical education can perhaps decrease the time it takes a novice to become an expert by accessing gaze patterns.

New frameworks for those studying and working in help are being developed by the international accreditation establishment to ensure the highest level of healthcare professionals. An example of such a framework is Competency-based medical education (CBME), which is aimed at ensuring that healthcare workers have high expertise that isn't just certified on paper but also proven by practise [79]. In recent years CBME is already being implemented by including new standards of teaching, assessment and curriculum into medical education and practice [80-84]. Due to these changes, we need new technology to facilitate the assessment procedure and eye-tracking in VR can surely become one of the major tools in this process.

Research in the field of medical education suggests that the pattern of eye movement of students changes with regards to diagnostics as they progress in the studies. More specifically, the eye movements become more rapid and move towards the important for diagnostic regions [78]. Hence, the eye-movement of students becomes more and more like that of an expert, this process can be time and analysed. It has also been suggested that this process can be accelerated by showing students video material of the expert's eye movement, this method is called eye-movement modeling examples (EMMEs).

The technology and application of EMMEs has been rapidly developing and many interesting studies have already been produced. EMMEs include video of expert eye movement as well as audio description of the action by the expert $[85,86]$. The scientific basis behind this method lies in neuroscience, where it has been shown that the brain can mirror actions, when another person's action is observed. Such a response is known as a "mirror system" and can be integrated into the learning process $[87,88]$. This technology provides students with the unique opportunity to learn from the experts in the field without them being physically available, this brings the home- or distant education to a new level of quality, which is particularly relevant during the pandemic outbreak [78].

EMMEs method had been used outside the medical education field, one of the studies reported that the use of EMMEs improved the ability of notice aircraft inspectors to detect aircraft faults [89]. The same has been observed for circuit board inspectors [90,91]. Moreover, EMMEs accelerated the speed 
of debugging for the software engineers [92]. Reports show that with the use of EMMEs students can become better readers and solve logical tasks such as puzzles faster [93].

When the gaze is analyzed with the use of eye-trackers, in the medical diagnostic field it is seen that a sequence of saccades and fixation over a medical image appears. In one study, radiographers viewed the eye movements of either fellow novices or experts during the learning process and then interpreted a chest X-ray [94]. Interestingly, it has been shown that the ability to notice and locate pulmonary conditions has improved in comparison to "free-search" not only from observing the experts but also from observing novices.

In a recent study, medical students observed a video of child epilepsy cases. The video was played in three different settings, in the first one the expert was narrating the video with voice, this was a control video sample. In the second, the eye-movement of the expert was traced onto the video by a small circle on the images. In the third video, the eye movements of the expert were also presented, but the area of the image, which the expert didn't focus on, was blurred out. The results of this study showed an increase in the diagnostic of students after viewing the third video and no change for the first or the second. Therefore, specific viewing conditions can facilitate the use of a "mirror" system in the brain and enhance the learning process [95].

The available research suggests that vision tracking in VR can be a useful tool for developing diagnostic skills in medical students. It is well known that the process of medical education and training is a long multi-step process, therefore, using eye-tracking in VR alongside conventional teaching techniques might be able to increase the efficiency and accelerate the training.

With the successful and effective implementation of eye-tracking in virtual reality into medical education and training, it makes sense to interpolate these findings into other fields of education. Based on the presented data, it can be argued that a field of scientific education, which usually involves a lot of lab work, would greatly benefit from the novelty of eye-tracking in virtual reality. The personalization of learning trajectories in chemistry education can be achieved in a variety of ways. The implementation of virtual reality can create an environment where students learn each topic at their own pace. Eye tracking can be used to collect the data regarding the process of a student on a chemical topic. From the available research, it can be seen, that the amount of time students spend on specific words or tasks, represents how well they know the topic. It can be anticipated that the better knowledge students have, the less time they will spend looking at incorrect answers and the quicker they will concentrate their gaze on the correct one in tests. Same assessment can be done with regards to chemical exercises. Naturally, some students will learn faster 
than others, and eye tracking could be a good measure of this process. The educational process can then be personalized, and the specific tasks where students spent the most time or couldn't concentrate their gaze on the correct answers, will be repeated. Specific topics on which extra work should be conducted will be highlighted for each student individually.

The use of eye tracking in virtual reality for conducting tests will eliminate the issue of cheating in tests or the absence of questions due to peer pressure and anxiety, since the results of such testing would be selfexplanatory for the teacher.

\subsection{Experimental set-up}

Experimental set-up of eye-tracking in VR includes both: hardware and software components. The hardware consists of a VR-set, which is the headmounted display that often comes with controllers. The controllers are held in hands and allow the user to interact with the virtual environment. The motion trackers that come with the VR-set are installed around the participant. The modern sets with high quality components can be provided include HTC Vive (https://www.vive.com/eu/product/), which has already been used for educational purposes. The eye-trackers can be purchased separately and are produced for specific types of headsets. There are also headsets that already include eye-trackers, for example HTC Vive Pro VR (https://www.vive.com/eu/product/vive-pro-eye/overview/). Hardware equipments also includes PC, which provides the interface for the use of VR headset. It is worth noting, that the PC has have powerful processors, therefore, the choice is often directed towards gaming PCs. Along with the PC, headphone sets and sets of cables are needed for each participant.

The software part includes licenses for tools, which allow to design and create virtual experiments. However, it is worth noting, that many instruments for VR are open source and available for free, such as the student version of game-engine Unity (https://unity.com/unity/features/vr). An example of a free tool that can be used for chemistry education is NarupaXR (https://irl.itch.io/narupaxr), which offers interactive molecular dynamic simulations in VR.

Although the complete set-up for eye-tracking in VR can seem quite costly, it is a necessary tool for modern classrooms and laboratories. The scope of applications and the advantages for the educational process outweigh the disadvantage of the price. Moreover, creating an infrastructure that can support distance and personalized learning is a challenge that educational systems in many countries are now facing, and virtual reality can be one of the high tech solutions for this issue. 


\section{Conclusions}

In conclusion, the current review has provided firstly an overview of the areas where the eye-tracking technology has been researched and implemented over the past decades. Secondly, it shows that the VR technology has been widely applied in many fields of scientific research over the past years and the interest in it continues to grow. It can be observed that both these technologies are sophisticated and well researched, moreover, we argue that now they can be used together and more extensively applied to the area of education. A major advantage of VR in the educational field is the ability to combine classic laboratory experience, while eliminating dangers that are associated with it for school and university students. Implementation of eye-tracking in VR provides new interesting approaches for studying the attention and motivation of students, possibly accelerating and making the education more efficient as well as a tool for assessment. The ability to use VR with different environments, model and control every aspect of the process, makes it an indispensable educational tool. In this review, we provide a possible trajectory for the development and application of these technologies in the classroom.

It is worth noting that development of new methods for using eyetracking in VR for education is especially relevant in the current situation, with many countries world-wide switching to distant teaching and the educational system trying to adapt to the changes. The methods of eyetracking in VR implementation with regards to hardware and software have been numerous reported, therefore this technology can soon become available in day to day life.

However, there are certain disadvantages for this technology, a major one of which being motion sickness and visual discomfort which appears while using VR for a longer time. It can prevent students from using the technology to its full potential or even using it at all. We believe that these obstacles will be surpassed due to the current extensive research in this field.

Overall, we believe that eye-tracking in combination with VR presents a powerful tool that can change the way we perceive education and greatly expand the potential of modern educational programs. This combination has already been successfully applied in the field of medical education, therefore we suggest that more people from different fields should pay attention to this technology and consider the possibility of its 
implementation into the educational process.

Funding: This work was supported by the Russian Science Foundation, grant no. 20-78-10135..

Conflicts of Interest: The authors declare no conflict of interest.

\section{References}

1. Rigby D., Vass C., Payne K. Opening the 'Black Box': An Overview of Methods to Investigate the Decision-Making Process in Choice-Based Surveys. Patient. 2020. Vol. 13, № 1. P. 31-41.

2. Brunyé T.T. et al. A review of eye tracking for understanding and improving diagnostic interpretation. Cogn. Res. Princ. Implic. 2019. Vol. 4, № 1.

3. How People Read Online: The Eyetracking Evidence. 2nd ed.

4. Cameirão M.S. et al. The impact of positive, negative and neutral stimuli in a virtual reality cognitive motor rehabilitation task: A pilot study with stroke patients. J. Neuroeng. Rehabil. 2016. Vol. 13, № 1.

5. Faria A.L. et al. Benefits of virtual reality based cognitive rehabilitation through simulated activities of daily living: a randomized controlled trial with stroke patients. J. Neuroeng. Rehabil. 2016. Vol. 13, № 1. P. 1-12.

6. Maggio M.G. et al. The Growing Use of Virtual Reality in Cognitive Rehabilitation: Fact, Fake or Vision? A Scoping Review. J. Natl. Med. Assoc. 2019. Vol. 111, № 4. P. 457-463.

7. Maggio M.G. et al. Cognitive rehabilitation in patients with traumatic brain injury: A narrative review on the emerging use of virtual reality. J. Clin. Neurosci. 2019. Vol. 61. P. 1-4.

8. Maggio M.G. et al. Virtual reality in multiple sclerosis rehabilitation: A review on cognitive and motor outcomes. J. Clin. Neurosci. 2019. Vol. 65. P. 106-111.

9. Peshkovskaya A.G. et al. The socialization effect on decision making in the Prisoner's Dilemma game: An eye-tracking study. PLoS One. 2017. Vol. 12, № 4.

10. Maggio M.G. et al. Virtual reality and cognitive rehabilitation in people with stroke: An overview. J. Neurosci. Nurs. 2019. Vol. 51, № 2. P. 101-105.

11. Brunyé T.T., Gardony A.L. Eye tracking measures of uncertainty during perceptual decision making. Int. J. Psychophysiol. 2017. Vol. 120. P. 60-68.

12. Spinks J., Mortimer D. Lost in the crowd? Using eye-tracking to investigate the effect of complexity on attribute non-attendance in discrete choice experiments Clinical decision-making, knowledge support systems, and theory . BMC Med. Inform. Decis. Mak. 2016. Vol. 16, № 1.

13. Nakhaeizadeh S. et al. The value of eye-tracking technology in the analysis and interpretations of skeletal remains: A pilot study . Sci. Justice. 2020. Vol. 60, № 1. P. 36-42.

14. Bault N., Wydoodt P., Coricelli G. Different Attentional Patterns for Regret and Disappointment: An Eye-tracking Study . J. Behav. Decis. Mak. 2016. Vol. 29, № 2-3. P. 194-205.

15. Helbing J., Draschkow D., Võ M.L.-H. Search superiority: Goal-directed attentional allocation 
creates more reliable incidental identity and location memory than explicit encoding in naturalistic virtual environments . Cognition. 2020. Vol. 196.

16. Fridman I., Ubel P.A., Higgins E.T. Eye-tracking evidence shows that non-fit messaging impacts attention, attitudes and choice . PLoS One. 2018. Vol. 13, № 10.

17. Smith S.M., Krajbich I. Gaze Amplifies Value in Decision Making . Psychol. Sci. 2019. Vol. 30, № 1. P. 116-128.

18. Pärnamets P. et al. Biasing moral decisions by exploiting the dynamics of eye gaze . Proc. Natl. Acad. Sci. U. S. A. 2015. Vol. 112, № 13. P. 4170-4175.

19. Rosa P.J. et al. Eye movement analysis and cognitive assessment: The use of comparative visual search tasks in a non-immersive vr application . Methods Inf. Med. 2017. Vol. 56, № 2. P. 112-116.

20. Reichenberger J., Pfaller M., Mühlberger A. Gaze Behavior in Social Fear Conditioning: An EyeTracking Study in Virtual Reality . Front. Psychol. 2020. Vol. 11.

21. Paletta L., Dini A., Pszeida M. Emotion Measurement from Attention Analysis on Imagery in Virtual Reality . Adv. Intell. Syst. Comput. 2020. Vol. 952. P. 13-20.

22. Piotrowski P., Nowosielski A. Gaze-Based Interaction for VR Environments . Adv. Intell. Syst. Comput. 2020. Vol. 1062. P. 41-48.

23. Orlosky J., Huynh B., Hollerer T. Using eye tracked virtual reality to classify understanding of vocabulary in recall tasks . Proceedings - 2019 IEEE International Conference on Artificial Intelligence and Virtual Reality, AIVR 2019. 2019. P. 66-73.

24. McNamara A., Jain E. Eye tracking and virtual reality . SIGGRAPH Asia 2019 Courses, SA 2019. 2019.

25. Huey E.B. The Psychology and Pedagogy of Reading. 1968. 469 p.

26. Buswell T. How Adults Read . Libr. Q. 1938. Vol. 8, № 3. P. 419-420.

27. Tatler B.W. et al. Yarbus, Eye Movements, and Vision . Iperception. 2010. Vol. 1, № 1. P. 7-27.

28. Levy-Schoen A. Eye movements and vision: A. L. Yarbus (translated by L. A. Riggs). Plenum Press, New York, 1967 XI+222 pp. \$17,50 . Neuropsychologia. 1968. Vol. 6, № 4. P. 389-390.

29. Hansen D.W., Ji Q. In the Eye of the Beholder: A Survey of Models for Eyes and Gaze . IEEE Trans. Pattern Anal. Mach. Intell. 2010. Vol. 32, № 3. P. 478-500.

30. Deubel H., Schneider W.X. Saccade target selection and object recognition: Evidence for a common attentional mechanism . Vision Res. 1996. Vol. 36, № 12. P. 1827-1837.

31. Holmqvist K., Nyström M., Mulvey F. Eye Tracker Data Quality: What It is and How to Measure It . Proceedings of the Symposium on Eye Tracking Research and Applications. New York, NY, USA: Association for Computing Machinery, 2012. P. 45-52.

32. Funke G. et al. Which Eye Tracker Is Right for Your Research? Performance Evaluation of Several Cost Variant Eye Trackers . Proc. Hum. Factors Ergon. Soc. Annu. Meet. 2016. Vol. 60, № 1. P. $1240-1244$.

33. Holmqvist K. et al. Eye Tracking: A Comprehensive Guide To Methods And Measures. 2011.

34. Eckstein M.K. et al. Beyond eye gaze: What else can eyetracking reveal about cognition and cognitive development? . Dev. Cogn. Neurosci. 2017. Vol. 25. P. 69-91.

35. Bird G., Press C., Richardson D.C. The role of alexithymia in reduced eye-fixation in autism 
spectrum conditions . J. Autism Dev. Disord. 2011. Vol. 41, № 11. P. 1556-1564.

36. Chawarska K., Macari S., Shic F. Context modulates attention to social scenes in toddlers with autism . J. Child Psychol. Psychiatry. 2012. Vol. 53, № 8. P. 903-913.

37. Shic F. et al. Limited activity monitoring in toddlers with autism spectrum disorder . Brain Res. 2011. Vol. 1380. P. 246-254.

38. Parish-Morris J. et al. Once Upon a Time: Parent-Child Dialogue and Storybook Reading in the Electronic Era . Mind, Brain, Educ. 2013. Vol. 7, № 3. P. 200-211.

39. Tenenbaum E.J. et al. Attention and word learning in autistic, language delayed, and typically developing children . Front. Psychol. 2014. Vol. 5, № MAY.

40. Klin A. et al. Visual fixation patterns during viewing of naturalistic social situations as predictors of social competence in individuals with autism . Arch. Gen. Psychiatry. 2002. Vol. 59, № 9. P. 809-816.

41. Norbury C.F. et al. Eye-movement patterns are associated with communicative competence in autistic spectrum disorders . J. Child Psychol. Psychiatry Allied Discip. 2009. Vol. 50, № 7. P. 834842.

42. Asan O., Yang Y. Using Eye Trackers for Usability Evaluation of Health Information Technology: A Systematic Literature Review . JMIR Hum. Factors. 2015. Vol. 2, № 1. P. e5.

43. Bond R.R. et al. Assessing computerized eye tracking technology for gaining insight into expert interpretation of the 12-lead electrocardiogram: an objective quantitative approach . J. Electrocardiol. 2014. Vol. 47, № 6. P. 895-906.

44. Krupinski E.A. Visual Search of Mammographic Images: Influence of Lesion Subtlety1 . Acad. Radiol. 2005. Vol. 12, № 8. P. 965-969.

45. Krupinski E.A., Graham A.R., Weinstein R.S. Characterizing the development of visual search expertise in pathology residents viewing whole slide images . Hum. Pathol. 2013. Vol. 44, № 3. P. 357-364.

46. Voisin S. et al. Predicting diagnostic error in radiology via eye-tracking and image analytics: Preliminary investigation in mammography . Med. Phys. 2013. Vol. 40, № 10. P. 101906.

47. Brunyé T.T. et al. Eye Movements as an Index of Pathologist Visual Expertise: A Pilot Study . PLoS One. Public Library of Science, 2014. Vol. 9, № 8. P. 1-7.

48. Mercan E. et al. Localization of Diagnostically Relevant Regions of Interest in Whole Slide Images: a Comparative Study . J. Digit. Imaging. 2016. Vol. 29, № 4. P. 496-506.

49. Nagarkar D.B. et al. Region of interest identification and diagnostic agreement in breast pathology . Mod. Pathol. 2016. Vol. 29, № 9. P. 1004-1011.

50. Barlinn J. et al. Brain Rehabilitation in Clinical Trials Setup by Eye-Tracking. 2016. P. 89-94.

51. Hollander J.B. et al. Seeing the city: using eye-tracking technology to explore cognitive responses to the built environment . J. Urban. Int. Res. Placemaking Urban Sustain. Routledge, 2019. Vol. 12, № 2. P. 156-171.

52. Poole A., Ball L.J. Eye tracking in HCI and usability research . Encyclopedia of Human Computer Interaction. 2005. 211-219 p.

53. Klein T.M., Drobnik T., Grêt-Regamey A. Shedding light on the usability of ecosystem services- 
based decision support systems: An eye-tracking study linked to the cognitive probing approach . Ecosyst. Serv. 2016. Vol. 19. P. 65-86.

54. Dupont L. et al. Analyzing the perception of water surfaces in urban landscapes using eyetracking. 2013.

55. De Lucio J. V et al. Visual landscape exploration as revealed by eye movement tracking. Landsc. Urban Plan. 1996. Vol. 34, № 2. P. 135-142.

56. Potocka I. The lakescape in the eyes of a tourist. Quaest. Geogr. 2013. Vol. 32, № 3. P. 85-97.

57. Kiefer P. et al. Eye tracking for spatial research: Cognition, computation, challenges . Spat. Cogn. Comput. 2017. Vol. 17, № 1-2. P. 1-19.

58. Torralba A. et al. Contextual guidance of eye movements and attention in real-world scenes: The role of global features in object search . Psychol. Rev. 2006. Vol. 113, № 4. P. 766-786.

59. Ehinger K.A. et al. Modelling search for people in 900 scenes: A combined source model of eye guidance . Vis. cogn. 2009. Vol. 17, № 6-7. P. 945-978.

60. Noland R.B. et al. Eye-tracking technology, visual preference surveys, and urban design: preliminary evidence of an effective methodology . J. Urban. 2017. Vol. 10, № 1. P. 98-110.

61. Kudelka V., Dobesova Z. Eye-tracking testing of GIS interfaces. 2015. Vol. 1. P. 585-592.

62. Clay V., König P., König S. Eye tracking in virtual reality . J. Eye Mov. Res. 2019. Vol. 12, № 1.

63. Radianti J. et al. A systematic review of immersive virtual reality applications for higher education: Design elements, lessons learned, and research agenda . Comput. Educ. 2020. Vol. 147. P. 103778.

64. Tanriverdi V., Jacob R.J.K. Interacting with eye movements in virtual environments . Conference on Human Factors in Computing Systems - Proceedings. 2000. P. 265-272.

65. Gergle D., Kraut R.E., Fussell S.R. Using visual information for grounding and awareness in collaborative tasks . Human-Computer Interact. 2013. Vol. 28, № 1. P. 1-39.

66. Hußmann H., Oechsner C. Gaze-based Interaction using Pursuits in VR environments. 2017.

67. Weiss S. et al. Applications of Immersive Virtual Reality in Nursing Education - A Review. 2018.

68. Pottle J. Virtual reality and the transformation of medical education . Futur. Healthc. J. Future Healthcare Journal, 2019. Vol. 6, № 3. P. 181-185.

69. Saxena N. et al. Virtual reality environments for health professional education . Cochrane Database Syst. Rev. 2016. Vol. 2016, № 2.

70. Celine G. et al. Eye-tracking in dentistry: What do children notice in the dentist? . J. Dent. 2018. Vol. 78. P. 72-75.

71. Merali N., Veeramootoo D., Singh S. Eye-Tracking Technology in Surgical Training . J. Investig. Surg. Taylor \& Francis, 2019. Vol. 32, № 7. P. 587-593.

72. Bergeron M., Lortie C.L., Guitton M.J. Use of Virtual Reality Tools for Vestibular Disorders Rehabilitation: A Comprehensive Analysis . Adv. Med. / ed. Todt I. Hindawi Publishing Corporation, 2015. Vol. 2015. P. 916735.

73. Rutkowski S. et al. Short-term exercise training in virtual reality in patients with COPD, solution or white elephant? . Eur. Respir. J. European Respiratory Society, 2019. Vol. 54, № suppl 63.

74. Ustun A., Yılmaz R., Karaoğlan Yılmaz F.G. Virtual Reality in Medical Education. 2020. P. 56-73. 
75. Hess E.H., Polt J.M. Pupil Size as Related to Interest Value of Visual Stimuli . Science (80-. ). American Association for the Advancement of Science, 1960. Vol. 132, № 3423. P. 349-350.

76. Lim Jia Zheng Jason Teo, J.M. Comparing Eye-Tracking versus EEG Features for Four-Class Emotion Classification in VR Predictive Analytics . Int. J. Adv. Sci. Technol. 2020. Vol. 29, № 6s SE-Articles. P. 1492-1497.

77. Pilgrim J.M., Pilgrim J. THE USE OF VIRTUAL REALITY TOOLS IN THE READINGLANGUAGE ARTS CLASSROOM . Texas J. Lit. Educ. 2016. Vol. 4. P. 90.

78. Jarodzka H. et al. In the eyes of the beholder: How experts and novices interpret dynamic stimuli . Learn. Instr. 2010. Vol. 20, № 2. P. 146-154.

79. Aggarwal R., Darzi A. Technical-skills training in the 21st century. The New England journal of medicine. United States, 2006. Vol. 355, № 25. P. 2695-2696.

80. Frank J.R., Danoff D. The CanMEDS initiative: implementing an outcomes-based framework of physician competencies. Med. Teach. Taylor \& Francis, 2007. Vol. 29, № 7. P. 642-647.

81. Nasca T.J. et al. The Next GME Accreditation System - Rationale and Benefits . N. Engl. J. Med. 2012. Vol. 366, № 11. P. 1051-1056.

82. Simpson J.G. et al. The Scottish doctor--learning outcomes for the medical undergraduate in Scotland: a foundation for competent and reflective practitioners . Med. Teach. Taylor \& Francis, 2002. Vol. 24, № 2. P. 136-143.

83. Swing S.R. The ACGME outcome project: retrospective and prospective . Med. Teach. Taylor \& Francis, 2007. Vol. 29, № 7. P. 648-654.

84. Holmboe E.S., Call S., Ficalora R.D. Milestones and competency-based medical education in internal medicine. JAMA Intern. Med. 2016. Vol. 176, № 11. P. 1601-1602.

85. Jarodzka H. et al. Learning to see: Guiding students' attention via a Model's eye movements fosters learning . Learn. Instr. 2013. Vol. 25. P. 62-70.

86. van Gog T. et al. Attention guidance during example study via the model's eye movements . Comput. Human Behav. 2009. Vol. 25, № 3. P. 785-791.

87. Calvo-Merino B. et al. Seeing or Doing? Influence of Visual and Motor Familiarity in Action Observation. Curr. Biol. Elsevier, 2006. Vol. 16, № 19. P. 1905-1910.

88. Calvo-Merino B. et al. Action Observation and Acquired Motor Skills: An fMRI Study with Expert Dancers. Cereb. Cortex. 2005. Vol. 15, № 8. P. 1243-1249.

89. Sadasivan S. et al. Use of Eye Movements as Feedforward Training for a Synthetic Aircraft Inspection Task. Proceedings of the SIGCHI Conference on Human Factors in Computing Systems. New York, NY, USA: Association for Computing Machinery, 2005. P. 141-149.

90. Nalanagula D., Greenstein J.S., Gramopadhye A.K. Evaluation of the effect of feedforward training displays of search strategy on visual search performance. Int. J. Ind. Ergon. 2006. Vol. 36, № 4. P. 289-300.

91. Stein R., Brennan S.E. Another Person's Eye Gaze as a Cue in Solving Programming Problems. Proceedings of the 6th International Conference on Multimodal Interfaces. New York, NY, USA: Association for Computing Machinery, 2004. P. 9-15.

92. Mason L., Pluchino P., Tornatora M.C. Eye-movement modeling of integrative reading of an 
illustrated text: Effects on processing and learning. Contemp. Educ. Psychol. 2015. Vol. 41. P. 172187.

93. Velichkovsky B.M. Communicating attention: Gaze position transfer in cooperative problem solving. Pragmat. \&amp; Cogn. John Benjamins, 1995. Vol. 3, № 2. P. 199-223.

94. Litchfield D. et al. Viewing another person's eye movements improves identification of pulmonary nodules in chest x-ray inspection. J. Exp. Psychol. Appl. 2010. Vol. 16. P. 251-262.

95. Jarodzka, H., Balslev, T., Holmqvist, K., Nyström, M., Scheiter, K., Gerjets, P., \& Eika, B. (2012)

Conveying clinical reasoning based on visual observation via eye movement modelling examples. Instructional Science, 40, 815-827. 